brain demonstrated deep white matter changes in the left parietal and posterior parietal/occipital regions and adjacent grey matter and two small punctate haemorrhages in the left midparietal region.

Case 2: Four week old male infant presented with recurrent focal seizures. No pyrexia or preceding symptoms were documented. EEG recorded two seizures with origin near the right midline with spread to right motor cortex and right hemisphere. MRI brain showed right deep white matter changes and left subcortical lesion.

Case 3: Eleven week old female infant admitted with pyrexia and lethargy. She had 6 episodes of febrile generalised tonic seizures over 48 hours, each less than 5 minutes in duration. EEG demonstrated some asymmetry and excess slow activities over right temporal region in sleep, with no epileptiform features. MRI brain was normal.

Case 4: Thirteen month old female presented with febrile status epilepticus, on a background history of pyrexia and diarrhoea for 3 days. EEG showed three epileptic spasms with background continuous epileptiform discharges, which were multifocal; predominantly right posterior and some frontal discharges were seen in sleep. MRI brain showed subtle high signal white matter changes in the frontal lobes bilaterally and multiple high signal areas throughout corpus callosum.

Discussion This case series demonstrates that HSV encephalitis should be considered in children with atypical febrile seizures which are prolonged, focal or recurrent. Timely recognition of HSE is essential to minimise patient morbidity and mortality.

\section{P507 TANACETUM PARTHENIUM, GRIFFONIA SIMPLICIFOLIA AND MAGNESIUM AS SYMPTOMATIC AND PROPHYLACTIC TREATMENT FOR HEADACHE IN PAEDIATRIC PATIENTS}

${ }^{1}$ Pietro Ferrara*, ${ }^{2}$ Federica Di Ruscio, ${ }^{3}$ Anna Rita Bellomo, ${ }^{4}$ Andrea lanni, ${ }^{4}$ Tommasangelo Petitti, Massimo Pettoello-Mantovani ${ }^{5}$. ${ }^{1}$ Institute of Pediatrics, Università Cattolica del Sacro Cuore, Rome, Italy; ${ }^{2}$ Campus Bio-Medico University, Rome, Italy; ${ }^{3}$ Giovan Battista Grassi Hospital, Rome, Italy; ${ }^{4}$ Public Health and Statistics, Campus BioMedico University, Rome, Italy; ${ }^{5}$ Department of Pediatrics, Scientific Institute Casa Sollievo della Sofferenza, University of Foggia, Foggia, Italy

\subsection{6/archdischild-2019-epa.843}

Objective The aim of the this study is to evaluate the use and the self-perceived efficacy and tolerability of three nutraceutical components - Tanacetum parteninum, Griffonia simpliciofila and Magnesium - in children and adolescents with primary headaches without other comorbidities.

Design This is a longitudinal cohort study.

Setting The study was conducted as a prospective study. After the first examination and the prescription of exams, patients were subjected to one month baseline period without treatment to verify the number and the intensity of attacks.

Patients We enrolled a total of 42 children, 25 female $(59,52 \%)$ and 17 male (40,48\%). The average age of children at the time of recruitment is $10,59 \pm 3,18$ years. The inclusion criteria employed have been: (a) aged between 3-17 years; (b) diagnosis of primary headache and/or migraine (including migraine with or without aura; chronic headache; persistent headache); (c) headache/migraine present for at least three months; (d) at least three migraine attacks per month in the last three months. The exclusion criteria have been: (a) patients with chronic pain of different nature; (b) secondary headache.
Interventions We administered AURASTOP $^{\circledast}$ at the dose of 1 teaspoon 2 times per day for 2-3 months and when an attack occurs, in children above 4 years of age, at a dose of 1 pouch.

Main outcome measures It has been used two validated questionnaire: Migraine Disability Assessment (MIDAS) and The six-item Headache Impact Test (HIT-6). MIDAS is a brief questionnaire and measures headache-related disability. HIT-6 was designed to provide a global measure of adverse headache impact. Treatment with AURASTOP ${ }^{\circledast}$ has been conducted for 3 months, with two sachets-day. Compliance of patients was monitored weekly by means of a weekly exchange of emails or phone calls to parents.

Results A reduction in MIDAS scores was observed comparing pre-treatment $(46.48 \pm 8.35)$ and post-treatment $(9.78 \pm$ 18.16) data. After the treatment $1 / 42$ patients $(2.38 \%)$ had worsened, 9/42 patients (21.43\%) had not improved, 10/42 (23.91\%) had gone up 1 class, $12 / 10$ patients $(28.57 \%)$ had gone up 2 classes, 10/42 (23.91\%) had gone up 3 classes of MIDAS score. Therefore, the improvement of disability related to the headache is statically significant (Fisher's exact test). The reduction of HIT-6 score post-treatment (46.48 \pm 8.35) compared with pre-treatment $(62.55 \pm 5.50)$ was statistically significant $(P<0.05)$.

Conclusions AURASTOP ${ }^{\circledast}$ can be used as a prophylactic treatment and as symptomatic treatment in paediatric patients with headache.

\section{P508 PARAECHOVIRUS MENINGOENCEPHALITIS IN INFANCY; A CASE REPORT}

'Laura Whitla*, 1,2,3 David Webb, 'Claire Purcell. 'Tallaght University Hospital, Dublin, Ireland; ${ }^{2}$ Our Lady's Children's Hospital Crumlin, Dublin, Ireland; ${ }^{3}$ Trinity College Dublin, Dublin, Ireland

\subsection{6/archdischild-2019-epa.844}

Aims This article aims to describe and highlight human paraechovirus (HPeV) as a cause of meningoencephalitis.

Methods A five week old (previously well) term male infant presented with a one day history of pyrexia and irritability. On examination, he was tachycardic, febrile and inconsolable. Baseline investigations showed serum white cell count (WCC) $4.5 \times 10^{9} / \mathrm{L}$ (46\% neutrophils, 37\% lymphocytes), CRP $11 \mathrm{mg} /$ L. Cerebrospinal fluid (CSF) analysis showed WCC $72 / \mathrm{cm}, 43 /$ $\mathrm{cm}$ and $114 / \mathrm{cm}$ in three samples (with $18 \%$ polymorphs and $82 \%$ mononuclear cells), protein $0.78 \mathrm{~g} / \mathrm{L}$, and glucose 3.3 $\mathrm{mmol} / \mathrm{L}$. Treatment with triple antibiotics and acyclovir was commenced pending further results. EEG on day 2 of admission was abnormal with low amplitude throughout and slow rhythmic activities. Due to ongoing severe irritability and persistent tachycardia, further investigations including an MRI brain and echocardiogram (ECHO) were also undertaken and were reassuringly normal. CSF and blood cultures were negative at 48 hours. However CSF polymerase chain reaction (PCR) result detected paraechovirus RNA; the CT value (crossing target) was 31.6 suggesting a relatively small viral load. CSF sampling was repeated 48 hours later and the WCC had improved to $15 / 54 / 38 \mathrm{cell} / \mathrm{cm}$. CSF PCR was again positive for paraechovirus and the CT value had increased to 39.2 suggesting a decrease in the viral load. This correlated with the slow clinical improvement. Of note the EEG on day 4 of admission was again very abnormal, as was the follow up EEG 6 weeks later which showed some excess slowing 
over the posterior regions but no epileptiform features. At most recent follow up review in outpatients at 5 months of age the patient is neurodevelopmentally normal with no notable sequelae from the significant episode of paraechovirus meningoencephalitis.

Conclusion $\mathrm{HPeV}$ causes a wide spectrum of diseases ranging from mild respiratory illnesses to severe life-threatening myocarditis and meningitis. Clinical symptoms, which are related to genotype and patient age, vary hugely. It is especially important to recognise $\mathrm{HPeV}$ (serotype 3 ), as it has the potential for significantly poor neurodevelopmental outcomes due to associated white matter changes (1). Prognosis must be guarded as, although it is a benign virus in the majority of cases, there are some important sequelae that parents need to be counselled about and clinical follow-up is essential to assess for any adverse neurodevelopmental outcomes.

\section{REFERENCES}

1. Britton PN, Dale RC, Nissen MD, Crawford N, Elliott E, Macartney K, et al. Parechovirus Encephalitis and Neurodevelopmental Outcomes. Pediatrics. 2016;137(2): e20152848.

\section{P509 POSTERIOR REVERSIBLE ENCEPHALOPATHY SYNDROME (PRES) IN A CHILD WITH STEROID-DEPENDENT NEPHROTIC SYNDROME: A CASE REPORT}

\begin{abstract}
${ }^{1,2}$ Anthea Bottoni* ${ }^{2}$ Felice Sica, ${ }^{1,2}$ Alessandra Basilone, 1,2Lorenza Chiossi, 1,2Barbara Damiano, 1,2Laura Ficele, ${ }^{2}$ Alessandra Marinari, 1,2Lucia Soldano, ${ }^{3}$ Massimo Pettoello-Mantovani, ${ }^{2}$ Angelo Campanozzi. ${ }^{1}$ Pediatric Residency Program, Foggia, Italy; ${ }^{2}$ Pediatrics, University Hospital of Foggia, Foggia, Italy; ${ }^{3}$ Pediatrics, Scientific Institute 'Casa Sollievo della Sofferenza', San Giovanni Rotondo (FG), Italy
\end{abstract}

10.1136/archdischild-2019-epa.845

Posterior reversible encephalopathy syndrome (PRES) is a clinico-radiological condition characterized by headache, nausea, vomiting, seizures, and visual disturbances with typical radiological features of symmetrical edema mostly involving the white matter in the occipital regions ${ }^{(1-2)}$. PRES can develop in association with a wide array of clinical conditions, including systemic infections, hypertension, organ transplantation, and immunosuppression (especially with calcineurin inhibitors). Children who are on prolonged steroid therapy or on calcineurin inhibitor therapy in nephrotic syndrome (NS) are at risk of developing PRES ${ }^{(3-4)}$.

A 6-year-old Moroccan-boy, followed in his country for nephrotic syndrome, was hospitalized in our unit of pediatrics for severe generalized body edema, proteinuria (18600 mg/ day), low serum albumin $(0.9 \mathrm{~g} / \mathrm{L})$, high serum cholesterol (468 mg/dl). His arterial blood pressure value was $122 / 77$ $\mathrm{mmHg}$. We treated him with steroid intravenous, albumin supplementations and we continued cyclosporine.

While his general conditions were improving, on the seventh day of hospitalization he developed headache, vomiting, dizziness, temporal blindness. A non-contrast computerized tomography was performed and it showed symmetrical hypodensities in parieto-occipital regions. His blood pressure was $132 / 71 \mathrm{mmHg}$. Then he developed two episodes of generalized tonic clonic convulsion, followed by unconsciousness. Magnetic resonance imaging (MRI) showed hyperintense signal in the parieto-occipital regions, and revealed bilateral cortical and subcortical white matter edema in parieto-occipital lobes

The child was treated with antihypertensive medications, diuretic, steroids and immunosuppressant (cyclosporine A), thereafter he got no seizure and regained full consciousness and vision. His blood pressure was kept at normal range and urinary protein excretion gradually decreased. MRI performed two weeks later revealed no abnormality of the brain, which is a usual phenomenon in case of PRES

The pathophysiology of PRES remains controversial, and two main hypotheses have been suggested; impaired cerebral autoregulation resulting in increased cerebral blood flow, and endothelial dysfunction with cerebral hypoperfusion ${ }^{(5-6)}$.

PRES must be managed carefully and its pathogenic factors should be suspected and recognized as soon as possible in order to properly treat the patient. In hypertension-related and drug-induced PRES, in fact, effective management includes prompt withdraw of offending agent, aggressive control of blood pressure, timely anti-convulsant therapy. In our case, hypertension was undoubtedly an important cause, but we were uncertain whether cyclosporine also played a pathogenic role.

PRES should be always considered in the differential diagnosis of a child with idiopathic nephrotic syndrome, headache and visual disturbance.

\section{P510 PARENTAL KNOWLEDGE OF CHILDREN'S SCREEN TIME AND THE DEPICTION OF NUTRITIONAL PRODUCTS ON CHILDREN'S TELEVISION}

Niall Dalton*, Katriona Fox, Madalene Khalil, Alex Boldy, Paul Scully, Clodagh O'Gorman. University Hospital Limerick, Limerick, Ireland

\subsection{6/archdischild-2019-epa.846}

Introduction Childhood obesity is a major risk factor for developing metabolic syndromes, with these patients five times as likely to develop type 2 diabetes compared to those without metabolic syndromes. Significant contributors to obesity include decreased physical activity, poor diet, and sedentary behaviours, especially television viewing. Current guidelines recommend no more than 2-hours non-educational screen-time per day.

Aims Examining parental knowledge regarding food-types on children's programming and ascertain self-reporting of television viewing and parental concerns regarding nutritional influence of television.

Methods Cross-sectional survey on parents of children aged 416 years old, presenting to University Hospital Limerick, October-April,2018. Surveys regarding demographics, television viewing, perceptions of television portrayal of nutrition. Data analysed on SPSS.

Results Sixty parents completed the surveys with 15\% reporting their children watched over 2 hours of television during weekdays, increasing 35\% during weekends. Whilst the majority (55\%) reported sweet snacks the most commonly depicted on television.

$10 \%$ of children always watched television during meals with half of children regularly watching television during meals. $80 \%$ of parents admitted concern regarding advertising of unhealthy foods with $85 \%$ doubting the advertising industry would protect children. $75 \%$ of parents were concerned regarding children nutrition, with various concerns expressed.

Conclusions Results showed high level of concern regarding advertising and children eating habits. Overall results showed significant proportion of children spending greater than the recommended time watching television, with a significant 